\title{
A detailed study of the rise phase of a long duration X-ray flare in the young star TWA 11B
}

\author{
J. López-Santiago ${ }^{1}$ and I. Crespo-Chacón ${ }^{1}$ \\ ${ }^{1}$ Departamento de Astrofísica y CC. de la Atmósfera, Universidad Complutense de Madrid, E-28040 \\ Madrid, Spain \\ and \\ G. Micela ${ }^{2}$ \\ ${ }^{2}$ INAF - Osservatorio Astronomico di Palermo Piazza Parlamento 1, I-90134 Palermo, Italy \\ and \\ F. Reale 3,2 \\ ${ }^{3}$ Dipartimento di Scienze Fisiche e Astronomiche, Sezione di Astronomia, Università di Palermo, Piazza \\ Parlamento 1, I-90134 Palermo, Italy
}

\begin{abstract}
We analyzed a long duration flare observed in a serendipitous XMM-Newton detection of the M star CD-39 7717B (TWA 11B), member of the young stellar association TW Hya ( 8 Myr). Only the rise phase (with a duration of $\sim 35 \mathrm{ks}$ ) and possibly the flare peak were observed. We took advantage of the high count-rate of the X-ray source to carry out a detailed analysis of its spectrum during the whole exposure. After a careful analysis, we interpreted the rise phase as resulting from the ignition of a first group of loops (event A) which triggered a subsequent two-ribbon flare (event B). Event A was analyzed using a single-loop model, while a two-ribbon model was applied for event B. Loop semi-lengths of $\sim 4 R_{*}$ were obtained. Such large structures had been previously observed in very young stellar objects $(\sim 1-4 \mathrm{Myr})$. This is the first time that they have been inferred in a slightly more evolved star. The fluorescent iron emission line at $6.4 \mathrm{keV}$ was detected during event B. Since TWA 11B seems to have no disk, the most plausible explanation found for its presence in the X-ray spectrum of this star is collisional- or photoionization. As far as we are concerned, this is only the third clear detection of Fe photospheric fluorescence in stars other than the Sun.
\end{abstract}

Subject headings: stars: activity — stars: coronae — stars: flare — stars: pre-main sequence — stars: individual (CD-39 7717B, TWA 11B)

\section{Introduction}

Among all the processes manifested in stellar coronae, flares are the most energetic ones. They are supposed to be the result of the energy release from magnetic field reconnection in the lower corona (e.g. Kopp \& Pneuman 1976). As a consequence, electrons and ions in the reconnection region are accelerated downwards, along the magnetic field lines, toward lower atmospheric layers.
When they reach the upper chromosphere, the local gas is heated and evaporated into the newformed magnetic loops. Thus, the density and temperature of these loops increase, causing intense emission of soft $(<10 \mathrm{keV})$ X-rays.

Stellar flares have been observed in almost all the H-R diagram (see Vaiana et al. 1981, for a review). However, they are more frequent in latetype stars, where they present a large variety of sizes and durations. In late- $\mathrm{K}$ and $\mathrm{M}$ dwarfs 
(the so-called UV Ceti-type stars; see Pettersen 1991, for a description of their main properties), moderate flares are frequently observed. During such events, the X-ray flux usually increases by a factor of $2-4$ (e.g. Robrade \& Schmitt 2005). Giant flares, in which the X-ray flux increases from dozens to hundreds times the quiescent state value, have been detected in some $M$ dwarfs such as EV Lac (Favata et al. 2000), EQ Peg (Katsova et al. 2002), and Prox Cen (Güdel et al. 2004). The duration of those flares are of the order of a few kiloseconds, even in the more energetic ones. For instance, the giant flare observed in $\mathrm{EV}$ Lac lasted $\sim 5 \mathrm{ks}$. Long-duration flares are more common in pre-main sequence stars than in the UV Ceti-type ones. For example, during the 13 days observing run of the ORION Nebula Complex by Chandra, at least 19 flares with durations above half a day were detected (Favata et al. 2005). Long-duration flares were also observed in the Taurus star-forming complex (Franciosini et al. 2007; Stelzer et al. 2007). The longer duration of these events is usually attributed to the presence of larger coronal structures in young stars.

The XMM-Newton and Chandra missions have contributed enormously to the understanding of the processes involved in the X-ray emission of late-type stars. In particular, the improved temporal and spectral resolution, together with the development of theoretical models (e.g. Kopp \& Poletto 1984; Poletto et al. 1988; Serio et al. 1991; Güdel et al. 1999; Reale et al. 2004; Reale 2007), have provided us with powerful tools for investigating coronal flares. Detailed diagnostics of X-ray flares have been carried out by different authors. Favata et al. (2005) determined general properties of flaring loops, in terms of temperature and semi-length, for young stellar objects in the Orion Nebula Complex (ONC). ¿From their analysis, the authors inferred loop semi-lengths comparable to the stellar radius in some cases. They speculated that these large structures are connected with the proto-planetary disk and are, in fact, the same structures that channel the plasma producing accretion. A similar work was done for the Taurus star-forming region (Franciosini et al. 2007), where the authors remarked that some coronal loops extended up to a distance comparable with the stellar radius (see also Giardino et al. 2004). Studies for more evolved stars were done by, e.g., Reale et al. (2004), Crespo-Chacón et al. (2007), and Testa et al. (2007), who found flaring loops with semi-lengths of $\sim 0.2-0.5 R_{\star}$ (i.e., a relatively compact flaring corona).

In the works presented in the previous paragraph, the diagnostic of X-ray flares was done using the procedure described by Reale et al. (1997, 2004) to model the decay phase, which assumes the flare to be produced in a single loop where heating does not entirely drive the flare decay. Reale (2007) extended this method to the rise phase and compared the parameters determined in this way with those obtained by analyzing the decay phase for three stellar flaring loops (see also Pan et al. 1997), finding a good agreement between them. On the other hand, the solar two-ribbon model developed by Kopp \& Poletto (1984), or its stellar version (Poletto et al. 1988), should be used when heating totally drives the flare evolution (Reale 2002, 2003). In this model, the reconnection energy is supposed to be dissipated immediately after being released. Kopp \& Poletto (1984) assumed that only a fraction of the magnetic energy released by the reconnection process is used to supply the thermal energy of the newly formed flare loops. Poletto et al. (1988) assumed that a factor of $10 \%$ of this thermal energy escapes into the $\mathrm{X}$-ray regime, as suggested by detailed studies of solar flares (Canfield et al. 1980). We also refer the reader to Güdel et al. (1999), who included time-dependent conductive and radiative losses in the X-ray corona self-consistently.

In this work, we analyze an XMM-Newton serendipitous observation of the young M-type star TWA 11B in which the X-ray emission suffered a continuous increase during approximately $35 \mathrm{ks}$. The duration and statistics of the observed rise have allowed us: (i) to carry out a detailed spectral time-analysis; and (ii) to derive properties of the star's magnetic configuration by using both the single-loop and the two-ribbon flare models described above.

\section{Observation and data treatment}

The XMM-Newton observation (ID 0006220201) was perfomed in the revolution 197, between 


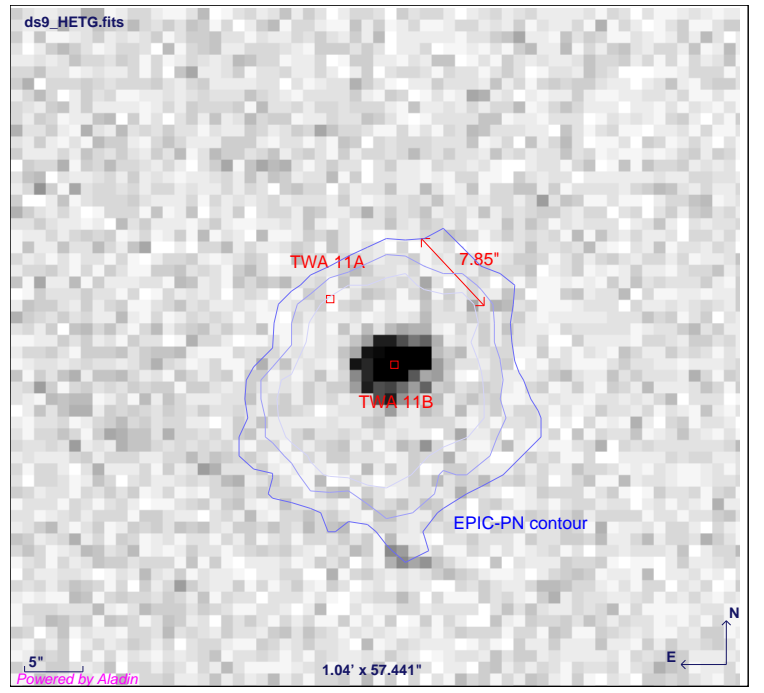

Fig. 1.- Chandra HETG serendipitous detection of TWA 11B. The position of the optical counterparts of TWA $11 \mathrm{~A}$ and TWA 11B are marked. Contours of the PSF observed in the XMM-Newton EPIC exposure are overplotted.

2001 January 4 and 5, for a total duration of $43 \mathrm{ks}$. The field is centred on the coordinates $\alpha_{2000}=12^{\mathrm{h}} 35^{\mathrm{m}} 34^{\mathrm{s}}$ and $\delta_{2000}=-39^{\circ} 54^{\prime} 55^{\prime \prime}$ (the main target being the Seyfert 2 galaxy NGC 4507). The EPIC cameras were operated in Full Frame mode using the Thick filter. Our target is situated 5.18 arcmin at the North-East of the central source, in the field of view of both the MOS and PN detectors. The EPIC-XMM source coincides with the position of the optical counterpart of TWA 11B. The primary star, TWA 11A (an A0type star), is situated at $\sim 8$ arcsec from TWA 11B. Although none X-ray emission is expected from an A0 star, we investigated the possibility that the primary star produced some X-rays that could affect our results. We studied a serendipitous detection of HETG Chandra of the source (Observation ID 2150). In this $140 \mathrm{ks}$ exposure Chandra observation, the X-ray source is clearly identified with TWA 11B (see Fig. 1), while none $\mathrm{X}$-ray emission coming from TWA $11 \mathrm{~A}$ is detected. Therefore, we are confident that the X-ray emission detected in the XMM-Newton observation comes only from the M star TWA 11B.

The data reduction followed the standard operating procedure. We used the version 7.1.0 of the
XMM-Newton Science Analysis System (SAS) to derive a table of calibrated events in the energy range $0.3-10.0 \mathrm{keV}$. To extract the events, we chose a radius of 30 arcsec, which is slightly larger than the $3 \sigma$-level of the source's PSF. This assures us to lose less than $1 \%$ of the counts from the source. Different filters were applied to eliminate bad events and noise. Note that the observation was neither affected by pile-up nor by high flaring background periods.

The X-ray light curve (Fig. 2) showed a total increase in the star's count-rate of a factor of 4.2 from the lower level $(\approx 0.25$ counts $/ \mathrm{s})$ to the maximum observed emission. Similar relative increases in flux were previously reported in flares from other stars (e.g. Robrade \& Schmitt 2005). A first increase in the light curve is observed $5 \mathrm{ks}$ after the beginning of the observation, reaching a local maximum only $5 \mathrm{ks}$ later. After a brief decrease, the count-rate continues increasing until reaching the global maximum in the light curve (this happened $37 \mathrm{ks}$ after the beginning and only $3 \mathrm{ks}$ before the end of the observation). The total duration of the enhancement is $32 \mathrm{ks}$ ( $\approx 9$ hours 1 ). Note that the observed maximum may or may not be the flare peak. In the latter case, the duration of the rise phase would be even longer.

In Fig. 3 we plot the evolution of the hardness ratio, which is a tracer of the temperature evolution, during the observation. Here, the soft energy band is defined as the range $0.3-0.8 \mathrm{keV}$ and the hard energy band as $0.8-4.5 \mathrm{keV}$. Fig. 3 shows that the mean coronal temperature reached a maximum at $t \sim 7 \mathrm{ks}$, maintained high with significant fluctuations during approximately the next $27 \mathrm{ks}$ of exposure, and then it began to decrease gradually.

Overall, the rise phase is unusually long compared with those observed in other stars (e.g. Pan et al. 1997; Güdel et al. 1999; Reale 2002; Robrade \& Schmitt 2005; Reale 2007; Crespo-Chacón et al. 2007). To date, so long rise phases have been observed only in some $\mathrm{T}$ Tauri stars (Favata et al.

\footnotetext{
${ }^{1}$ The rotation period of TWA $11 \mathrm{~B}$ is not known. Scholz et al. (2007) measured a projected rotational velocity $v \sin i=12.11 \pm 0.93 \mathrm{kms}^{-1}$, what leads to an upper limit in the rotational period of 2.7 days. Thus, the observation cover, at least, $17 \%$ of the rotational period of the star. Nevertheless, no indication of occultation of the flaring region is observed.
} 


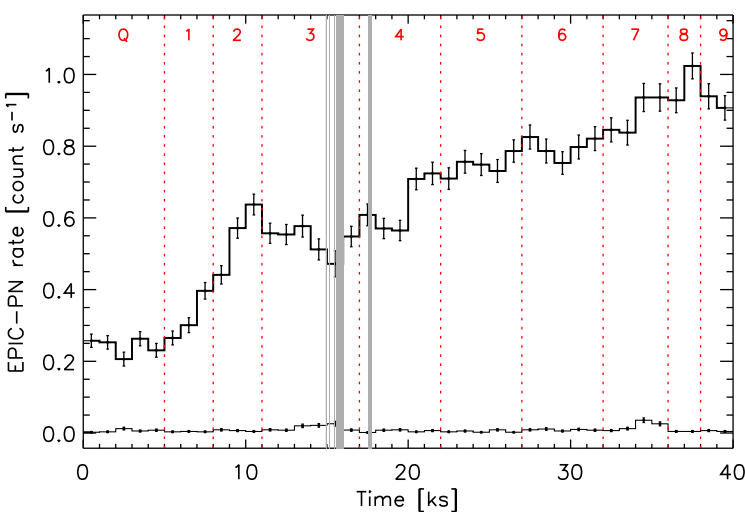

Fig. 2.- EPIC-PN light curve of TWA 11B in the energy range $0.3-10.0 \mathrm{keV}$. The curve was binned to a $1 \mathrm{ks}$ time resolution. The event list was corrected of bad events and noise. The exposure was also corrected of live-time and good-time intervals. The gray segments mark the periods in which the intrument was turned off. The continuous line at the bottom is the background light curve. The time blocks used in the spectral analysis are plotted as dashed vertical lines.

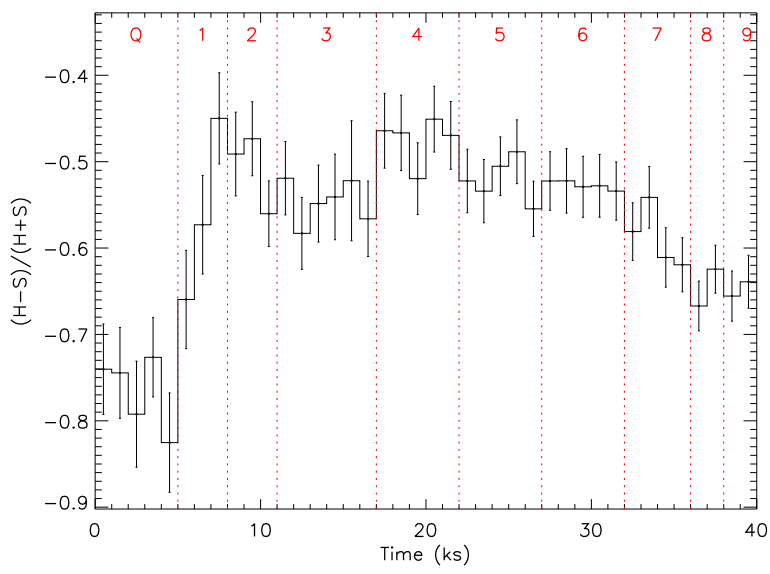

Fig. 3.- Hardness ratio evolution of TWA 11B during the observation with EPIC-PN. The time blocks used in the spectral analysis are plotted as in Fig. 2 for clarity.

2005; Giardino et al. 2006; Franciosini et al. 2007), some of them with accretion disks, and several RS CVn systems (Testa et al. 2007; Nordon \& Behar 2007). In such stars, the relative increase in flux ranges from a factor 3 (V410 Tau; Franciosini et al.
2007) to a factor 10 (V892 Tau; Giardino et al. 2004) with rise-phase times from 8 to $55 \mathrm{ks}$. TWA $11 \mathrm{~B}$ is a weak-line T Tauri M2.5 star with an age of $\sim 8 \mathrm{Myr}$ (estimated from its membership in the TW Hya Association). While it shows no signatures of an accretion disk (near-infrared color excess or strong $\mathrm{H} \alpha$ emission; Stauffer et al. 1995), it is still contracting. Thus, physical conditions in its atmosphere should be more similar to those of sub-giant stars than of main-sequence ones. This may be the reason why long duration rise phase flares are observed in both T Tauri stars and RS CVn systems.

A more detailed inspection of the light curve shows that the emission increased faster initially, it had a well-defined local maximum at $t \approx 10 \mathrm{ks}$, and then it growed again, but more gradually this time, until the end of the observation. At the very end, there is a hint that the emission was stopping to increase. Such a long rise phase suggests that we observed an uninterrupted sequence of flare events involving an extended coronal region. However, the faster initial rise and the local emission peak - coupled to the earlier hardness ratio peak - resembles the evolution of a self-standing flaring episode possibly occurring (at least during these initial stages) in a single loop (e.g. Reale 2007), whose decay merged with the ignition of the later overlapping events. Fig. 4 demonstrates that this peak is indeed significative and cannot be attributed to the effect of noise in the light curve. This figure shows the cumulative distribution of counts for 200 simulated constantly increasing light curves (for $t \geq 5 \mathrm{ks}$ ) with Poissonian noise (shadowed region) together with the cumulative distribution of counts observed during the rise (continuous line). Clearly, the simulations do not reproduce the observed distribution of counts for the first peak in the light curve. The differences are high enough to justify the treatment of this event as independent. In the following, we work under this hypothesis.

The large number of counts collected from the source in the EPIC cameras allowed us to divide the observation in several time intervals with enough signal to perform a reliable spectral analy-

\footnotetext{
${ }^{2}$ Constantly increasing light curves have been chosen for the simulations since the global enhancement mostly shows a linear pattern.
} 


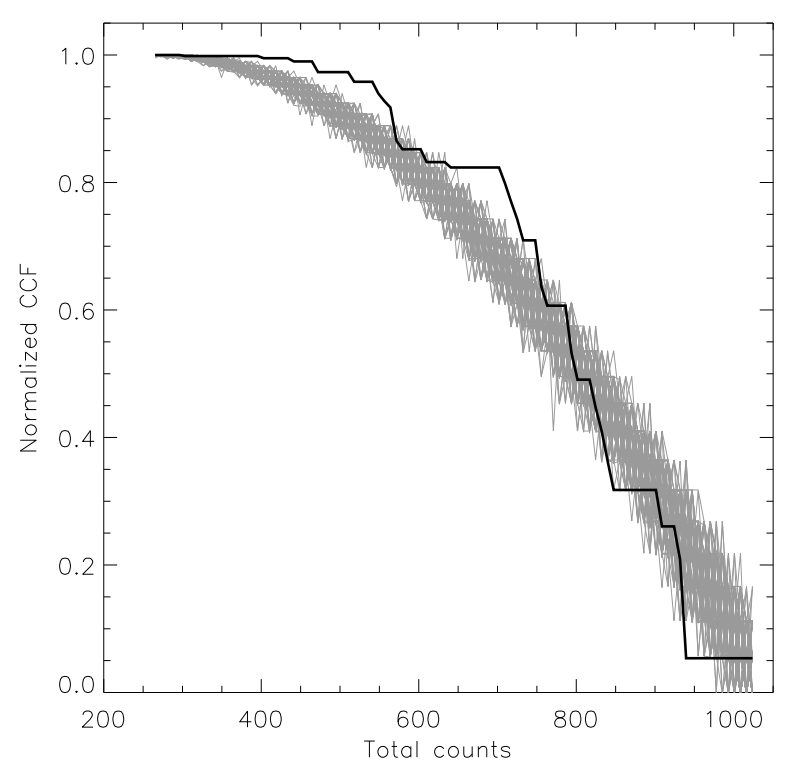

Fig. 4. - Normalized cumulative distribution of counts in the light curve of TWA $11 \mathrm{~B}$ for $t \geq 5 \mathrm{ks}$ (continuous line). The shadowed region contains cumulative curves of 200 simulated constantly increasing light curves (also for $t \geq 5 \mathrm{ks}$ ) with Poissonian noise.

sis $(\geq 1000$ counts in the quiescent and intervals 1 and 2 , and $\geq 2000$ counts in the remaining intervals after background subtraction 3 ). This permitted: 1) to investigate the nature of the processes taking place in our target; and 2) to derive physical properties of it.

\section{Spectral analysis}

For the spectral analysis, the observation was split into 10 time-intervals (vertical dashed lines in Fig. 2). These intervals sample different features in the light curve. We used the XSPEC spectral fitting package (Arnaud 1996, 2004) in the PN, MOS1, and MOS2 detectors simultaneously. We adopted the Astrophysical Plasma Emission Code (APEC, Smith et al. 2001a) included in the XSPEC software. APEC calculates spectral models for hot, optically thin plasmas using the Astrophysical Plasma Emission

\footnotetext{
${ }^{3}$ Intervals 1 and 2 have been studied separately to investigate the evolution of the parameters of the flaring plasma in its very first phases.
}

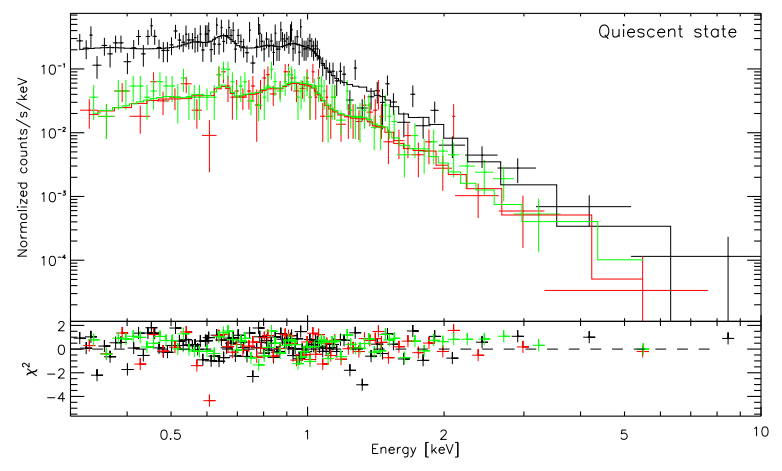

Fig. 5.- Observed EPIC PN and MOS spectra of TWA 11B during the quiescent state (marked with $\mathrm{Q}$ in Fig. 2).

Database (APED, Smith et al. 2001b), that contains the relevant atomic data for calculating both the continuum and line emission. Interstellar absorption was taken into account using the interstellar photo-electric absorption cross-sections of Morrison \& McCammon (1983), also available in XSPEC.

\subsection{Quiescent state}

The lowest (constant) count-rate level, found at the beginning of the observation, is assumed to be the quiescent state. The X-ray luminosity of the star during this lapse of time (assuming the same distance than the primary $d=67 \pm 3$ $\mathrm{pc})$ is $\log L_{\mathrm{X}}\left[\mathrm{erg} \mathrm{s}^{-1}\right]=29.35$, corresponding to $\log \left(L_{\mathrm{X}} / L_{\mathrm{bol}}\right)=-3.1$. These values are typical of both pre-main sequence stars members of young stellar associations (e.g. Kastner et al. 2003) and older field M dwarfs showing high X-ray activity (Robrade \& Schmitt 2005; Crespo-Chacón et al. 2007). The best fit to the X-ray quiescent spectrum (Fig. 51) is given by a plasma model with two equally-weighted thermal components (the output parameters of this fit are summarized in Table 1). Although high uncertainties are obtained for the hydrogen column density in our fit, the value of $N_{\mathrm{H}}$ obtained by us is very similar to that determined for other members of the TW Hya Association (e.g. Stelzer \& Schmitt 2004; Argiroffi et al. 2005). Besides, the interstellar extinction was found to be negligible in the general direction of TW Hya (Rucinski \& Krautter 1983). 
Table 1: Output parameters from fitting the Xray EPIC spectra of the quiescent state with a $2 T$-model.

\begin{tabular}{lll}
\hline$N H$ & $=1.7_{-1.7}^{+2.6} \times 10^{20} \mathrm{~cm}^{-2}$ \\
$Z$ & $=0.17_{-0.06}^{+0.08} Z_{\odot}$ \\
$k T_{1}$ & $=0.27_{-0.03}^{+0.03} \mathrm{keV}$ \\
$E M_{1}$ & $=2.2_{-0.8}^{+1.4} \times 10^{52} \mathrm{~cm}^{-3}$ \\
$k T_{2}$ & $=0.98_{-0.08}^{+0.08} \mathrm{keV}$ \\
$E M_{2}$ & $=2.2_{-0.5}^{+0.6} \times 10^{52} \mathrm{~cm}^{-3}$ \\
$\chi_{\text {red }}^{2}$ (d.o.f.) & $=0.86(176)$ \\
\hline
\end{tabular}

\section{2. $\quad$ Flaring state}

Synthesized stellar-like spectra of solar flares (previously subtracted by the quiescent "background" spectrum) are generally well-fitted with a single thermal component (1- $T$ model, see Reale et al. 2001). Consequently, to analyze physical properties of the flaring plasma in our observation, we first subtracted the spectrum of the quiescent state from the observed spectrum in each time-interval. Then we fitted a $1 T$-model, leaving its temperature $\left(k T_{\mathrm{F}}\right)$ and emission measure $\left(E M_{\mathrm{F}}\right)$ as free parameters. The values of the column density $(\mathrm{NH})$ and abundance $(\mathrm{Z})$ were fixed to those derived for the quiescent state. With this technique, we interpret that the coronal spectrum during the flare results from adding to the quiescent spectrum a third thermal component, which is ascribed to heated material filling the flaring loops (Reale et al. 2001; Crespo-Chacón et al. 2007). Models with additional temperature components were checked, but did not improve the fit results significantly.

Results from spectral fitting are given in Table 2. As expected from the hardness ratio evolution (Fig. 3), the temperature peaked in timesegment 1 . Then, it decreased gradually but almost continuously until the end of the observation. On the other hand, the emission measure increased continuosly from the beginning of the rise and starts to decrease only near the end of the observation.

\subsubsection{Evolution of the emission measure distri- bution}

In order to approximate physically more realistic continuous emission measure distributions (EMDs) of the plasma, we used a multitemperature model as in Robrade \& Schmitt (2005). In that work, the authors used a $6 T$ model on a logarithmic, almost equidistant grid with temperatures fixed at $0.2,0.3,0.6,1.2,2.4$, and $4.8 \mathrm{keV}$ (which correspond to $2.3,3.5,7.0$, 14.0, 28.0 and 56.0 MK), sampling those spectral regions where the XMM-Newton detectors are more sensitive. To fit our spectra, the values of $N H$ and $Z$ were fixed to those previously determined for the quiescent state. Thus, the only variables are the emission measures. Note that in this case we are fitting each spectrum as a whole (i.e., the quiescent spectrum was not subtracted from the rest of the spectra). Results from applying this $6 T$-model to our observation are shown in Table 3 and Fig. 6, and are summarized in the following items:

- The amount of plasma emitting at temperatures above $28 \mathrm{MK}$ is negligible in the quiescent state.

- Plasma emitting at the highest temperatures ( $\sim 56 \mathrm{MK})$ appears at the beginning of the first (faster) rise (time-segment 1), while the rest of the EMD curve remains as in the quiescent state. Note that in this time-segment we obtained the maximum temperature for the flaring component when fitting it with the $1 T$-model (see first part of $\S 3.2$ and Table 21).

- The only significant difference between the EMD of the quiescent state and that derived for time-segment 2 (where the light curve of the first flare peaks) is an excess of plasma emitting at temperatures around $28 \mathrm{MK}$. We interpret it as the cooling of the material at higher temperatures that was detected in time-segment 1 together with additional plasma evaporated after the temperature peak was reached in time-segment 1 (note that the total emission measure of the flaring component in time-segment 2 is higher than that measured in time-segment 1 , as it can also be seen in Table 2). 
Table 2: Spectral results for the flaring component in each time-segment of the light curve (excepting the quiescent time-interval).

\begin{tabular}{cccccc}
\hline \hline Time-segment & $\begin{array}{c}\text { Time-interval } \\
(\mathrm{ks})\end{array}$ & $\begin{array}{c}\text { Central time } \\
(\mathrm{ks})\end{array}$ & $\begin{array}{c}k T_{\mathrm{F}} \\
(\mathrm{keV})\end{array}$ & $\begin{array}{c}E M_{\mathrm{F}} \\
\left(10^{52} \mathrm{~cm}^{-3}\right)\end{array}$ & $\chi_{\text {red }}^{2}$ (d.o.f.) \\
\hline 1 & $5-8$ & 6.5 & $8_{-4}^{+60}$ & $0.72_{-0.17}^{+0.20}$ & $1.05(129)$ \\
2 & $8-11$ & 9.5 & $2.4_{-0.3}^{+0.4}$ & $4.0_{-0.3}^{+0.3}$ & $0.87(215)$ \\
3 & $11-17$ & 14 & $2.5_{-0.3}^{+0.4}$ & $3.88_{-0.28}^{+0.27}$ & $1.08(369)$ \\
4 & $17-22$ & 19.5 & $2.27_{-0.27}^{+0.25}$ & $5.3_{-0.3}^{+0.3}$ & $0.87(355)$ \\
5 & $22-27$ & 24.5 & $1.88_{-0.14}^{+0.14}$ & $7.2_{-0.3}^{+0.3}$ & $0.89(406)$ \\
6 & $27-32$ & 29.5 & $1.66_{-0.08}^{+0.18}$ & $8.3_{-0.3}^{+0.3}$ & $1.03(430)$ \\
7 & $32-36$ & 34 & $1.31_{-0.05}^{+0.05}$ & $9.2_{-0.4}^{+0.4}$ & $0.97(379)$ \\
8 & $36-38$ & 37 & $1.22_{-0.06}^{+0.06}$ & $9.9_{-0.5}^{+0.5}$ & $1.01(243)$ \\
9 & $38-41$ & 39.5 & $0.99_{-0.04}^{+0.04}$ & $9.7_{-0.4}^{+0.4}$ & $0.86(313)$ \\
\hline
\end{tabular}

Table 3: Output parameters (emission measures, in units of $10^{52} \mathrm{~cm}^{-3}$ ) from fitting the whole X-ray EPIC spectra to a $6 T$-model with temperatures fixed at $0.2,0.3,0.6,1.2,2.4$ and $4.8 \mathrm{keV}$. $E M_{1}^{\prime}$ is the emission measure corresponding to the thermal component of $0.2 \mathrm{keV}, E M_{2}^{\prime}$ is that of $0.3 \mathrm{keV}$, and so on. Uncertainties are calculated for a $2.7 \sigma$ confidence level.

\begin{tabular}{ccccccc}
\hline \hline Time Interval & $E M_{1}^{\prime}$ & $E M_{2}^{\prime}$ & $E M_{3}^{\prime}$ & $E M_{4}^{\prime}$ & $E M_{5}^{\prime}$ & $E M_{6}^{\prime}$ \\
\hline Quiescent & $1.00_{-0.91}^{+0.88}$ & $1.00_{-1.00}^{+1.28}$ & $0.77_{-0.59}^{+0.61}$ & $1.63_{-0.28}^{+0.26}$ & $0.00_{-0.00}^{+\ldots . .}$ & $0.00_{-0.00}^{+0.09}$ \\
1 & $0.66_{-0.66}^{+0.80}$ & $0.59_{-0.59}^{+1.51}$ & $0.75_{-0.75}^{+0.63}$ & $1.85_{-0.72}^{+0.63}$ & $0.00_{-0.00}^{+1.04}$ & $0.85_{-0.30}^{+0.29}$ \\
2 & $0.13_{-0.13}^{+1.24}$ & $2.14_{-2.14}^{+0.96}$ & $0.31_{-0.31}^{+1.15}$ & $2.12_{-0.97}^{+1.15}$ & $3.70_{-2.17}^{+0.66}$ & $0.00_{-0.00}^{+1.23}$ \\
3 & $1.31_{-1.09}^{+0.61}$ & $0.22_{-0.22}^{+1.58}$ & $2.04_{-0.78}^{+0.48}$ & $2.73_{-0.60}^{+0.54}$ & $0.00_{-0.00}^{+0.61}$ & $1.91_{-0.28}^{+0.29}$ \\
4 & $0.85_{-0.84}^{+0.98}$ & $1.88_{-1.88}^{+0.88}$ & $0.01_{-0.01}^{+0.92}$ & $4.48_{-0.88}^{+0.92}$ & $1.32_{-1.32}^{+1.86}$ & $1.48_{-1.08}^{+1.39}$ \\
5 & $0.54_{-0.54}^{+1.07}$ & $2.42_{-2.11}^{+2.40}$ & $0.62_{-0.62}^{+1.02}$ & $5.19_{-0.60}^{+1.17}$ & $1.69_{-1.69}^{+1.89}$ & $1.41_{-1.07}^{+1.06}$ \\
6 & $0.92_{-0.92}^{+1.20}$ & $1.94_{-1.94}^{+1.14}$ & $0.96_{-0.96}^{+1.04}$ & $5.41_{-1.22}^{+1.08}$ & $2.53_{-2.17}^{+1.01}$ & $1.01_{-1.01}^{+1.22}$ \\
7 & $1.26_{-1.24}^{+1.46}$ & $1.36_{-1.36}^{+2.32}$ & $1.30_{-1.25}^{+1.06}$ & $8.35_{-1.49}^{+1.24}$ & $0.86_{-0.86}^{+1.75}$ & $0.58_{-0.58}^{+0.82}$ \\
8 & $2.64_{-2.64}^{+1.17}$ & $0.25_{-0.25}^{+4.07}$ & $3.39_{-2.00}^{+1.01}$ & $8.17_{-2.07}^{+1.38}$ & $0.09_{-0.09}^{+2.40}$ & $0.81_{-0.81}^{+0.60}$ \\
9 & $1.38_{-1.38}^{+1.32}$ & $0.83_{-0.83}^{+2.90}$ & $4.08_{-1.66}^{+1.08}$ & $8.39_{-1.02}^{+0.98}$ & $0.00_{-0.00}^{+0.82}$ & $0.23_{-0.23}^{+0.40}$ \\
\hline
\end{tabular}

- The excess of plasma emitting in the region around $28 \mathrm{MK}$ that was observed in timesegment 2 continues cooling towards lower temperatures during time-segment 3 . At the same time, a high quantity of plasma appears again at the highest temperatures $(\sim$ $56 \mathrm{MK})$. This quantity is even larger than that measured in time-segment 1 and is approximately coincident with the beginning of the second (more gradual) rise.
- During time-segments $4-7$, the amount of plasma emitting at the highest temperatures remains still high, while a large excess of material (compared to the quiescent state) appears also between 7 and $28 \mathrm{MK}$ (reaching the maximum always at a temperature $\approx 14 \mathrm{MK})$. The existence of intense, sustained heating is needed to explain the maintenance of plasma at temperatures around $56 \mathrm{MK}$. The evolution of the rest of the EMD curve can be interpreted as the cooling of the 


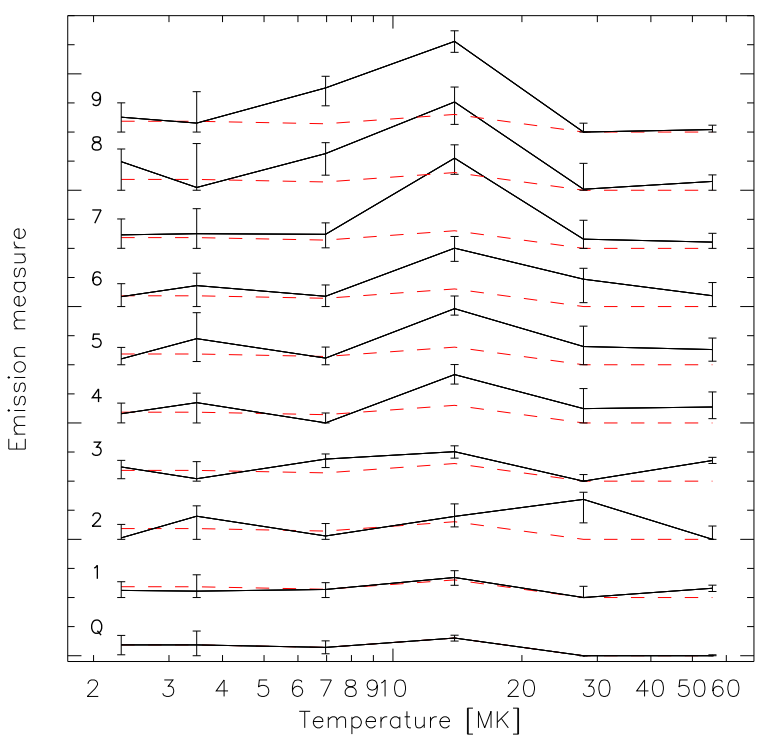

Fig. 6.- EMDs as derived with the $6 T$-model. The curves are shifted in the y-axis to better observe the flare evolution in time. Each one of the EMDs (shown as continuous lines) is labeled with the same number as its corresponding timesegment (see Table 2). The quiescent is marked as Q. For comparison, the EMD of the quiescent state is also overplotted as a red, dashed line at the same zero level than each one of the rest EMDs.

continuously new appearing very hot plasma together with heated material that evaporates from lower layers and fills the flaring loops.

- During time-segments 8 and 9 the emission at temperatures $\gtrsim 28 \mathrm{MK}$ turns back to the level found at the quiescent state, which is an indication of the strong heating not being present any more. However, the height of the maximum in the EMD curve is even larger than in the previous time-segments, appearing also excess emission at even lower temperatures (down to $\sim 3 \mathrm{MK}$ ).

\section{Flare modeling}

Results from $\S 2$ and $\S 3$ support the idea of the observed rise $(t \geq 5 \mathrm{ks})$ being the consequence of two separate flare events: the first more impulsive one (event A) starting in timesegment 1 , followed by a more gradual energy release (event B) having a beginning that is merged with the decay phase of event $\mathrm{A}$ and becoming dominant at the end of time-segment 3. But, what kind of magnetic structures are producing each one of these events? According to Reale (2007), multiple loop structures can be involved in a flare, but this frequently occurs only in its late phases. The initial phases of an X-ray flare are usually quite localized and one can reasonably assume the presence of a single dominant loop (e.g. Aschwanden \& Alexander 2001; Reale et al. 2004). This assumption is realistic enough in most of the observed rise phases, where the impulsive heating typically involves a dominant loop (while later residual heating may be released in other similar adjacent loops). The clear evidence of a delay between the temperature peak and the density peak is consistent with a single loop model, at least until the moment in which this maximum density is reached. This delay is often observed both in solar flares (e.g. Svlwester et al. 1993), and in stellar flares (e.g. van den Oord et al. 1988; van den Oord \& Mewe 1989; Favata et al. 2000; Maggio et al. 2000; Stelzer et al. 2002), even in very long ones (Favata et al. 2005; Getman et al. 2008). The presence of this delay is indeed a signature of a relatively short heat pulse (the larger the delay, the shorter the heat pulse is) and of the coherent hydrodynamic evolution of plasma confined in a loop. Arcades and two-ribbon flares are instead characterized by strong and/or lasting heating (Kopp \& Poletto 1984) and/or irregular light curves (Aschwanden \& Alexander 2001; Reale et al. 2004). Thus, event A is likely characterized (or dominated) by a single flaring loop since its ignition occurs only at time-segment 1 (as suggested by the plasma appearing at very high temperatures in its corresponding EMD, see $\S$ 3.2.1) and shows a delay between the temperature peak and the density peak reached by the flaring plasma in it (see Table 2). Event B is probably triggered by event A. The evolution of the whole EMD during event B evidences continuous, but decreasing in efficiency, heating of material and is consistent with event B being a tworibbon flare. In $\S 4.2$ we demonstrate that the light curve of event B is indeed well-reproduced by a two-ribbon flare model. Continual reconnections 
in an arcade may also be responsible for event B. However, in this case one would expect a more irregular pattern in the light curve than that observed in Fig. 2, and the EMD evolution would not necessarily point to a kind of heating that is decreasing in efficiency with time, as we have observed.

\subsection{Event A: the single loop flare}

We used the results from fitting the spectra with the $1 T$-model (see $\S 3$ ), given in Table 2, to determine the length of the loop involved in event A. For the temperature in time-segment 1, we used $k T=4 \mathrm{keV}$ (the lower limit from the fit) since its upper limit is undetermined (see Table 2).

For this event, the complete rise phase is observed and instead we miss satisfactory information from the decay phase. A complete theoretical analysis of the rise phase of a flare occurring in a single loop is given by Reale (2007)). The author showed that the loop half-length $(L)$ can be determined from the maximum temperature reached by the flaring plasma $\left(T_{0}\right)$ and the temperature and time in which the density peaks $\left(T_{\mathrm{M}}\right.$ and $t_{\mathrm{M}}$, respectively) by:

$$
L \approx 3 \cdot 10^{5 / 2} t_{\mathrm{M}} \frac{T_{0}^{5 / 2}}{T_{\mathrm{M}}^{2}}
$$

where all the parameters are given in c.g.s. units. The maximum temperature $\left(T_{0}\right)$ is related to the temperature measured for the flaring component by spectral fitting $\left(T_{\text {obs }}\right)$. For the EPIC instrument:

$$
T_{0}=0.13 T_{\mathrm{obs}}^{1.16}
$$

For event $\mathrm{A}$, the maximum temperature and maximum emission measure were reached in the time-segments 1 and 2, respectively. For a single loop description, the (square root of the) emission measure becomes a good proxy of the density, because the loop volume presumably does not change much during the event. In this case, from Eq. 11 we obtain a loop half-length $L=$ $1.8 \pm 0.3 \times 10^{11} \mathrm{~cm}$, i.e. $\sim 4 \pm 1 R_{\star}$ (assuming a stellar radius $R_{\star}=0.64 R_{\odot}$ - from the pre-main sequence models of Siess et al. 2000, for an M2.5 star with $8 \mathrm{Myr}$, such as TWA $11 \mathrm{~B}$ is supposed to be). Although this value is relatively large,
Table 4: Parameters of the flaring loop involved in event A, derived as in Reale (2007). We have assumed a semi-circular geometry.

\begin{tabular}{llc}
\hline \hline Parameter & \multicolumn{1}{c}{ Units } & Value \\
\hline$n_{\mathrm{M}}$ & $10^{10} \mathrm{~cm}^{-3}$ & $2.0 \pm 0.7$ \\
$n_{\text {avg }}$ & $10^{10} \mathrm{~cm}^{-3}$ & $4.1 \pm 0.7$ \\
$V$ & $10^{31} \mathrm{~cm}^{3}$ & $2.3 \pm 0.8$ \\
$A$ & $10^{20} \mathrm{~cm}^{2}$ & $0.6 \pm 0.2$ \\
$r$ & $10^{10} \mathrm{~cm}$ & $0.4 \pm 0.1$ \\
$L$ & $10^{11} \mathrm{~cm}$ & $1.8 \pm 0.3$ \\
\hline
\end{tabular}

similar loop sizes have already been derived for young stars in star-forming regions (Favata et al. 2005; Franciosini et al. 2007). As far as we are concerned, this would be the first time that such a relatively long loop is detected in a star older than 3 - 4 Myr.

This loop would fill a volume $V \approx 2.3 \times 10^{31} \mathrm{~cm}^{3}$ (see Reale 2007, for further details on the relations used for determining this quantity). Assuming a semi-circular geometry, its aspect $(r / L$, with $r$ being the loop cross-section) would be of the order of $2 \%$, which is quite lower than that observed in the Sun $(\sim 10 \%)$, but compatible with the results in Favata et al. (2005). In Table 4 we summarize the main parameters of the loop involved in event A, i.e. those already mentioned and maximum density at the loop apex $\left(n_{\mathrm{M}}\right)$, average density in the loop when the maximum density is reached at the apex $\left(n_{\text {avg }}\right)$ and cross-section area of the loop $(A)$. Such parameters were determined with the relations given in Reale (2007).

Comparing our results with those obtained by Reale (2007) for events observed in Algol, AB Dor, and Prox Cen, we conclude that: 1) our densities seem to be similar to those determined in the Algol and Prox Cen flares; and 2) the loop volume and its subtended area are of the same order of magnitude as those found for Prox Cen, another $\mathrm{M}$ star. Note that the largeness of some of the errors shown in Table 4 is a consequence of the propagation of errors in the equations.

For the sake of completeness, we made the exercise to repeat the same analysis to the entire flare event $(\mathrm{A}+\mathrm{B})$ as if it all occurred in a single 
loop. For a flare temperature peaking in timesegment $1\left(t_{0} \approx 6.5 \mathrm{ks}\right)$, and the density peaking in time-segment $8\left(t_{\mathrm{M}} \approx 37 \mathrm{ks}\right)$, using Eq. 1. we obtained $L=1.3 \times 10^{13} \mathrm{~cm}$, i.e. $L \sim 260 R_{\star}$. Obviously, such a long loop would be easily destroyed by the stellar rotation. Clearly, this result makes no sense.

\subsection{Event B: the two-ribbon flare}

For the study of event B, we used the tworibbon flare model by Kopp \& Poletto (1984) extended to the stellar case (Poletto et al. 1988). This model supposes that a disruptive event opens a loop arcade, being the open field lines then driven toward a radial neutral sheet (above the magnetic neutral line) where they reconnect at progressively higher altitudes. Thus, the continuous heating provided by these reconnections is capable of reproducing the temporal profile of the energy rate released during both the rise and decay phases of a two-ribbon flare. By analogy to that observed on the Sun, the model considers that the arcade of loops is extended along the East-West direction (i.e., axial symmetry around the polar axis is assumed). It also assumes that the magnetic field is potential between the stellar surface and the location of the neutral line and extends radially outwards from there. The magnetic field in the meridional planes of the arcade can therefore be expressed in terms of a single lobe of a Legendre polynomial of degree $n$. Note that:

i Each lobe is latitudinally bounded by radial magnetic fields.

ii The arcade corresponds to one lobe axisymmetrically continued over some longitude in the East-West direction.

iii Through an appropriate choice of $n$, one can find a lobe placed in the range of latitudes covered by the active region. However, as spatial information is available only for the Sun, stellar flaring regions are generally assumed to be centered on the equator for odd $n$ and to end at the equator for even $n$.

iv As stellar observations cannot provide any information on the time-dependent rise of the neutral point, it is assumed to mimic the solar case. Thus, it follows an exponential law of the form given by Eq. 3, where $y$ is the height of the neutral point (in units of $R_{\star}$, measured from the star's center), $t$ is the time (measured from the beginning of the two-ribbon flare, $\left.t_{\text {ini }}\right), t_{0}$ is a timeconstant, and $H_{\mathrm{m}}$ is the maximum height reached by the reconnection point during its upward movement (measured from the star's surface). $H_{\mathrm{m}}$ is typically chosen to be equal to the latitudinal extent of the arcade, which is in turn linked with $n$ (see Eqs. 4 and 5 ).

$$
\begin{array}{rlrl}
y & =1+\frac{H_{\mathrm{m}}}{R_{\star}}\left(1-e^{-\frac{t}{t_{0}}}\right) & & \\
H_{\mathrm{m}} & \approx \frac{\pi}{n+1 / 2} R_{\star} & & \text { for } n>2 \\
H_{\mathrm{m}} & \approx \frac{\pi}{2} R_{\star} & \text { for } n=2
\end{array}
$$

Under all these assumptions, the rate of magnetic energy released by the reconnecting arcade per radian of longitude $(d E / d t)$ can be expressed as:

$$
\begin{aligned}
\frac{d E}{d t} & =\frac{1}{8 \pi} 2 n(n+1)(2 n+1)^{2} R_{\star}^{3} B_{\mathrm{m}}^{2} \\
& \times \frac{I_{1,2}(n)}{P_{n}^{2}\left(\theta_{1,2}\right)} \frac{y^{2 n}\left[y^{2 n+1}-1\right]}{\left[n+(n+1) y^{2 n+1}\right]^{3}}\left(\frac{d y}{d t}\right)
\end{aligned}
$$

where $I_{1,2}(n)=\int P_{n}^{2}(\theta) \mathrm{d}(\cos \theta)$ evaluated between the latitudinal borders of the lobe, $P_{n}(\theta)$ is the Legendre polynomial of degree $n, \theta$ is the co-latitude, $\theta_{1,2}$ is the co-latitude of either boundary of the lobe, and $B_{\mathrm{m}}$ is the maximum surface magnetic field in the active region. The factor $B_{\mathrm{m}}^{2}$ merely defines the normalization of the energy release light curve, while $t_{0}$ and $n$ determine its shape. As Eq. [6 is given per radian of longitude, a length $(l)$ must be assumed for the arcade in order to calculate its total energy-release rate. Solar two-ribbon flares occur in loop arcades whose length is typically about 1.5 times their width $\left(l \approx 1.5 H_{\mathrm{m}}\right)$. In our study, we adopted this ratio for any given $n$.

Since, in the view of many authors, the initiation process for the flare itself might be the result of a more rapid (nearly explosive) reconnection than the reconnection process about which Kopp \& Poletto (1984) and Poletto et al. (1988) speak, they stressed that their model is applicable 
only after the initial flare trigger mechanism is terminated. In fact, the model seems not to be able to describe the time of impulsive heating and steeply increasing temperatures. However, the impulsive phase typically ceases before reaching the $50 \%$ flare peak level in the soft X-ray bandpass commonly used for stellar observations. At this time in our observations, the temperature is gradually decreasing. Hence, the model is applicable from time-segment 4 of the light curve to the end of our observations (time-segment 3 is rejected also for avoiding possible contamination from the decay of event A).

In order to apply the described model, the photospheric magnetic field in the flaring region (from which $B_{\mathrm{m}}$ can be determinated), and its latitudinal location and size (which dictate $n$ ) should be known. In the solar case, observations provide all these data, whereas in the stellar case, at best, they can only be inferred indirectly. Since we are unaware of the location, size, and magnetic field strength of the active region that we are studying, we treated $n$ and $B_{\mathrm{m}}$ as free parameters to be determined from the best fit of the model to the observations.

We created a grid of values for the free parameters $n, t_{0}$, and $t_{\text {ini }}$ to fit event $\mathrm{B}$ with the two-ribbon flare model (the lower limit of $t_{\text {ini }}$ was fixed at the beginning of the whole enhancement because of obvious physical reasons). For each set of these parameters, we determined the $B_{\mathrm{m}}$ that best fit the data by minimizing the $\chi^{2}$ value, which is defined as:

$$
\chi^{2}=\sum_{i=1}^{N}\left(\frac{L_{\mathrm{mod}, i}-L_{\mathrm{obs}, i}}{\Delta L_{\mathrm{obs}, i}}\right)^{2}
$$

where $L_{\text {mod }}$ is the expected luminosity from the model $\left(L_{\text {mod }}=f \cdot q \cdot l \cdot d E / d t\right), L_{\text {obs }}$ is the observed luminosity, $\Delta L_{\mathrm{obs}}$ is the error in the observed luminosity, and $N$ the number of time-intervals with which we fitted the two-ribbon model (from time-segment 4 to 9 ). Following the solar analogy (Canfield et al. 1980, see $\S$ 1), we assumed the measured radiative losses in the X-ray band to be $\approx 10 \%$ of the thermal energy generated as consequence of magnetic reconnections, that is $f \approx 0.1$ (see Poletto et al. 1988, for details). Actually, only a fraction $(q<1)$ of the liberated magnetic energy is indeed used to heat the plasma (thermal energy that is subsequently lost via radiation and conduction), while the rest is transformed into mechanical energy, into fast particles ejected from the corona, etc. At this point, we want to notice that we did not find a unique solution corresponding to a single set of parameters, but a number of solutions producing a good fit $\left(\chi^{2} \sim 1\right)$.

In Table 5, we show the two-ribbon flare parameters for some of the good fits we have obtained. We plot these results together with the observations in Fig. 7. Other good fits were found also for larger values of $n$ (i.e., active regions with smaller width and, therefore, shorter loops), but note that a smaller active region needs higher surface magnetic fields for reproducing a given energy-release rate. Thus, loop systems that reach larger altitudes - i.e., small values of $n$ - may be more realistic in our case, although other loop configurations cannot be excluded (see $\S$ 政).

Table 5: Two-ribbon flare parameters resulting from fitting the model to the observed energy release rate from event $B$.

\begin{tabular}{lcccc}
\hline Polynomial degree & 2 & 3 & 5 & 10 \\
Region width [deg] & $90^{\circ}$ & $53^{\circ}$ & $33^{\circ}$ & $17^{\circ}$ \\
$H_{\mathrm{m}}\left[\mathrm{R}_{\star}\right]$ & 1.57 & 0.90 & 0.57 & 0.30 \\
$L_{\mathrm{m}}^{\dagger}\left[\mathrm{R}_{\star}\right]$ & 2.46 & 1.41 & 0.89 & 0.47 \\
$t_{\mathrm{ini}}[\mathrm{ks}]$ & 7.0 & 6.5 & 6.0 & 5.5 \\
$t_{0}[\mathrm{ks}]$ & 247 & 192 & 183 & 174 \\
$B_{\mathrm{m}} \sqrt{q}[\mathrm{G}]$ & 440 & 730 & 1050 & 1830 \\
$\mathrm{v}_{\mathrm{rise}}\left[\mathrm{km} \mathrm{s}{ }^{-1}\right]$ & 2.8 & 2.1 & 1.4 & 0.8 \\
$N_{\mathrm{e}}\left[\times 10^{10} \mathrm{~cm}^{-3}\right]$ & 1.1 & 2.6 & 5.1 & 13.3 \\
$\chi^{2}$ & 1.02 & 1.02 & 1.04 & 1.04 \\
\hline & is the loop semi-length determined from the maximum \\
\multicolumn{4}{c}{ height $H_{\mathrm{m}}$ assuming a semi-circular geometry. }
\end{tabular}

The values given in Table 5 for the maximum surface magnetic field were determined assuming that all the magnetic energy is used to heat the plasma that fills the flaring loops $(q=1)$. Kopp \& Poletto (1984) used $q=0.003$ for a solar flare. On the other hand, during the analysis of a stellar flare, Güdel et al. (1999) found acceptable solutions with $q<0.05$ for $n=2$ and $q \approx 0.01-0.02$ for $n=3-4$. For event $\mathrm{B}$ we found that small values of $q(\sim 0.01-0.02)$ imply very strong photospheric magnetic fields in the flaring region $\left(B_{\mathrm{m}} \approx 3-18 \mathrm{kG}\right)$. Using $q=0.05$, we obtained $B_{\mathrm{m}} \approx 2 \mathrm{kG}$ for $n=2$ and $B_{\mathrm{m}} \approx 3-8 \mathrm{kG}$ 


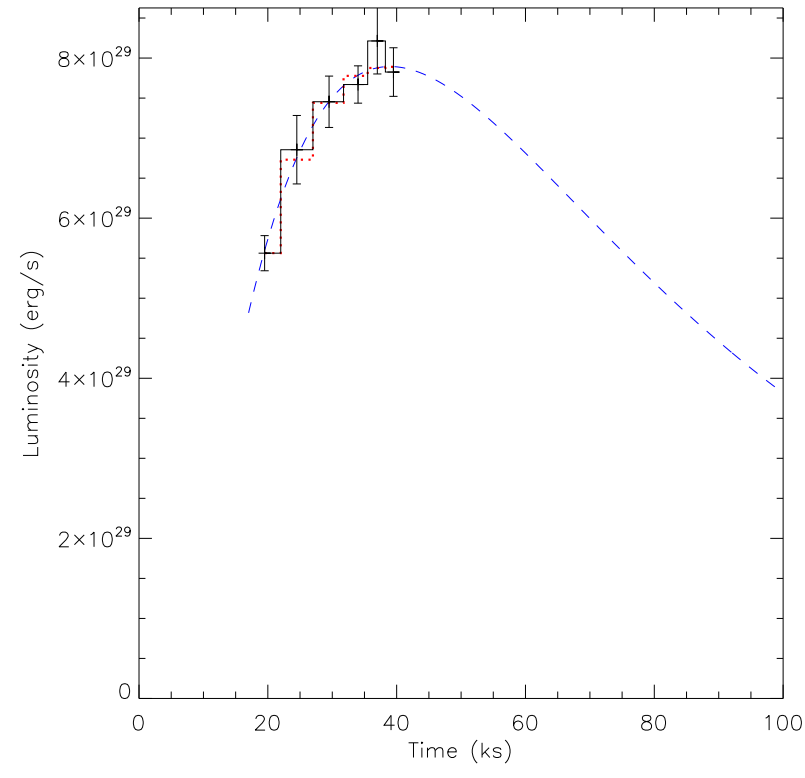

Fig. 7.- Best fit to the observations using the two-ribbon model for $n=2$ (see Table 5). Note that each set of parameters in Table 5 produces similar results in the fitted region while having different slopes in the previous part and in the decay. The light curve segments used to fit the tworibbon model (time-segments 4-9, see also Fig. 2) is shown with a solid line. The dashed line is the model. We have also plotted the average values of the model in each time-interval during event B (dotted line) for a clearer comparison between the observed light curve and the model.

for $n=3-10$.

\section{The fluorescent Fe $6.4 \mathrm{keV}$ line}

During the inspection of the X-ray spectrum in the different time-intervals, we observed a feature (excess emission not reproduced by the plasma model) close to the $\mathrm{Fe} \mathrm{K}_{\alpha}$ line at redder wavelengths. We identified this feature as the Fe fluorescent line at $6.4 \mathrm{keV}$. It was noticeable only during the time-segments 4 to 9 , while it was neither in time-segments 1 to 3 nor in the quiescent state (see Fig. 8). Unfortunately, we could not monitor the time-variation of this emission line since the individual spectra of each time-interval have no counts enough for accurately fitting the line.
Table 6: Best fitting values (3T plasma model + Gaussian) for the spectrum integrated in the timesegments $4-9$. Only the temperature and the emission measure of the third thermal component and the Gaussian are listed because the remaining parameters were fixed to the values estimated for the quiescent state. The given $\chi_{\text {red }}^{2}$ and d.o.f. values refer to the total fit.

\begin{tabular}{lll}
\hline$k T_{3}(\mathrm{keV})$ & $=2.02_{-0.12}^{+0.13}$ \\
$E M_{3}\left(10^{52} \mathrm{~cm}^{-3}\right)$ & $=4.7_{-0.3}^{+0.3}$ \\
Gaussian central energy $(\mathrm{keV})$ & $=6.4_{-0.6}^{+0.9}$ \\
Gaussian flux $\left(10^{-6} \mathrm{ph} \mathrm{cm}^{-2} \mathrm{~s}^{-1}\right)$ & $=0.9_{-1.3}^{+0.7}$ \\
Gaussian $\sigma(\mathrm{eV})$ & $=9.7_{-9.7}^{+0.6}$ \\
$\chi_{\text {red }}^{2}$ [d.o.f.] & $=0.96[499]$ \\
\hline
\end{tabular}

Thus, to quantify the excess emission at $6.4 \mathrm{keV}$ in our observations, we performed spectral fitting using an interstellar absorbed 3T-plasma model and an additional Gaussian line component at $6.4 \mathrm{keV}$. We fitted, on the one hand, the spectrum integrated from the quiescent to time-segment 3 (inclusive) and, on the other hand, the spectrum integrated from time-segment 4 to 9 (inclusive). The gaussian flux obtained in this way for the former spectrum is zero. The best fit results are shown in Table 6. Note that we left free only the temperature and the emission measure of the third (hottest) thermal component and the gaussian, while the rest of the parameters were fixed to the values obtained for the quiescent state (Table 1).

In the past, fluorescent Fe emission were commonly observed in classical T Tauri stars and protostars (Tsuiimoto et al. 2005; Favata et al. 2005; Giardino et al. 2007; Sciortino 2008), where it has been attributed to the incidence of X-ray emission onto the proto-planetary disk or into the circumstellar gas surrounding very young objects. However, the Fe $6.4 \mathrm{keV}$ line has been observed also in the giant star HR 9024 (Testa et al. 2008) and the RS CVn system II Peg (Osten et al. 2007). In the case of HR 9024, the authors attributed its presence to the incidence of hard X-rays onto the photosphere, in concordance with what is observed in the Sun. In II Peg, the excitation mechanism was ascribed to electron impact ionization of photospheric Fe.

In our observation, we have not statistics 

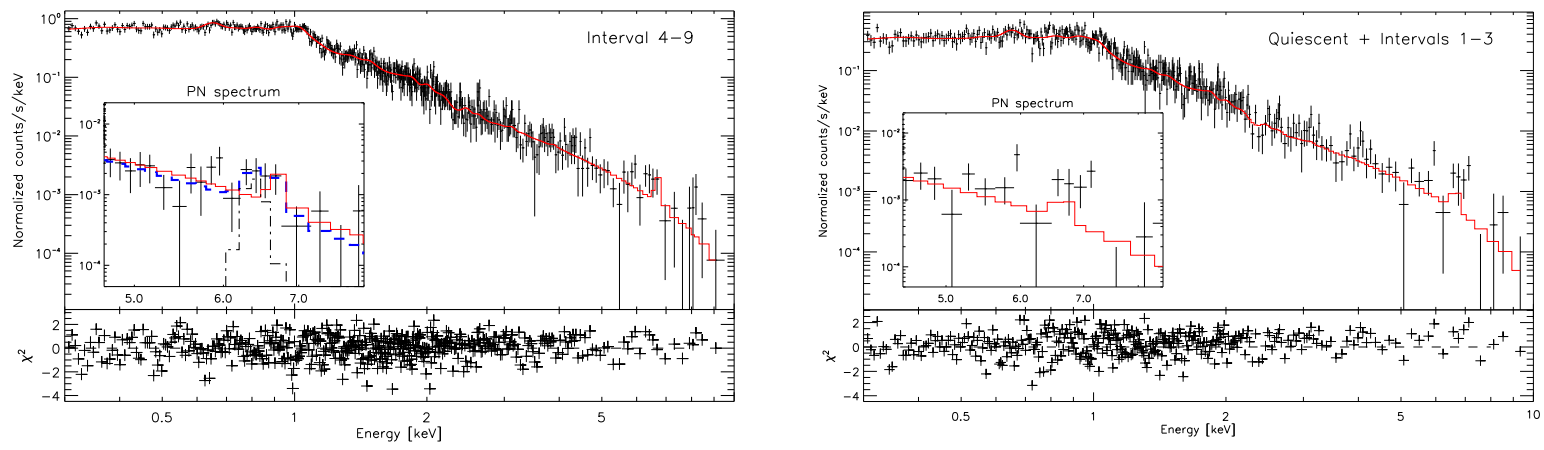

Fig. 8.- Left: PN spectrum of TWA 11B integrated in the time-segments $4-9$, with the clear excess emission in $6.4 \mathrm{keV}$. The (red) continuous line is the fitted $3 T$-model. In the small window, the (blue) dashed-line is the fitted 3T-model + Gaussian component. The Gaussian component is also plotted as a dotted-dashed line. Right: Same as figure at the left but in the time-segments $1-3$ plus quiescent. Here, the excess emission in $6.4 \mathrm{keV}$ is clearly not present.

enough to perform a robust analysis of the fluorescent line. Nevertheless, some constraints can be given. On the one hand, the line is not observed during the first event. This may be consistent with the scenario of the long loop for this event. The efficiency of the fluorescence decreases with the distance of the X-ray source to the photosphere (e.g. Drake et al. 2008). Therefore, if the fluoresce line were produced by photoionization, it should not be observed during flares occurring in long loops. On the other hand, the presence of the line during the second event could be indicative of a not very high loop system, if it were produced by photoionization. In contrast, if the line were produced by collisional ionization, there would be none constraint to the loop height. A result in favor with the non-photoionization nature of the fluorescence line is shown in Czesla \& Schmitt (2007). In their study, the authors modeled the illuminating input spectrum and obtained line fluxes below the observations. With our data, the collisional production of the fluorescence line cannot be discarded.

\section{Final remarks and conclusions}

In this paper, we analyzed the long rise phase of a flare observed in an XMM-Newton archive data of the $\sim 8$ Myr old star TWA 11B. The analysis of the light curve, of the hardness-ratio curve, and of the time-resolved spectra consistently indicates that probably the flare first involved mainly a single loop and then propagated to a loop arcade becoming a proper two-ribbon flare. We split our analysis into three parts: the quiescent state, the single-loop flare (event A), and the two-ribbon system (event B). Event A was studied with the analysis described in Reale (2007). For event B, we used the stellar version of the Kopp \& Poletto (1984)'s solar two-ribbon flare model that is able to provide some limited information about the late flaring structures.

For the single loop, we obtained a semi-length of approximately $1.8 \pm 0.3 \times 10^{11} \mathrm{~cm}\left(\sim 4 \pm 1 \mathrm{R}_{\star}\right)$, with a volume $V=2.3 \times 10^{31} \mathrm{~cm}^{3}$ and a crosssection $r / L \sim 2 \%$. These values are comparable to those found by Favata et al. (2005) in Orion members. This fact makes us suggest that large and thin loops are common in young active stars. For the two-ribbon system, different results consistent with the observed data were found. Good fits $\left(\chi^{2} \sim 1\right)$ were obtained for both small and large values of $n$ (i.e. for high and short loop arcades). Bearing the semi-length of the first loop in mind, the more realistic scenario for the two-ribbon system is that with long loops. In any case, the estimated values of the maximum surface magnetic field in the flaring region result to be quite strong, reaching $2-8 \mathrm{kG}$ when a large fraction of the liberated magnetic energy $(q=0.05)$ is assumed to heat the plasma, and up to $18 \mathrm{kG}$ when $q \sim 0.01$.

During the inspection of the X-ray spectrum, we observed the Fe fluorescent line at $6.4 \mathrm{keV}$ 
during the time in which the two-ribbon system evolved (time-segments $4-9$ ). The absence of the line during the first event may be consistent with the long loop scenario for event A. In contrast, the detection of the fluorescence line during the second event could be indicative of a not very high loop system involved in the two ribbon flare, if the line were produced by photoionization. Otherwise, there would be none constraint to the loop system height.

JLS acknowledges financial support by the PRICIT project S-0505/ESP-0237 (ASTROCAM) of the Comunidad Autónoma de Madrid (Spain). ICC acknowledges support from the Spanish Ministerio de Educación y Ciencia, under the grant F.P.U. AP2001-0475; and from the Marie Curie Actions grant MERG-CT-2007-046535. The Madrid group acknowledges partial support by the Programa Nacional de Astronomía y Astrofísica of the Spanish Ministerio de Educación y Ciencia (MEC), under grants AYA2008-00695 and AYA2008-06423-C03-03. FR acknowledges support from Italian Ministero dell'Università e della Ricerca (MIUR). GM acknowledges support from the Agenzia Spaziale Italiana (ASI) and the Istituto Nazionale di Astrofisica (INAF) under grant ASI-INAF I/088/06/0. We would like to thank the referee and the editor for useful comments and discussion that helped us to improve this manuscript.

\section{REFERENCES}

Argiroffi, C., Maggio, A., Peres, G., Stelzer, B., \& Neuhäuser, R. 2005, A\&A, 439, 1149

Arnaud, K. 1996, XSPEC: The First Ten Years. In ASP Conf. Ser. 101, eds. George H. Jacoby and Jeannette Barnes, 17

Arnaud, K. 2004, XSPEC: Progress and Plans. In the American Astronomical Society, HEAD meeting $8,16.29$

Aschwanden, M. J., \& Alexander, D. 2001, Sol. Phys., 204, 91

Canfield, R. C., Cheng, C.-C., Dere, K. P., et al. 1980, In Solar flares, ed. Sturrock P. A., p. 451

Crespo-Chacón, I., Micela, G., Reale, F., et al. 2007, A\&A, 471, 929
Czesla, S., \& Schmitt, J. H. H. M. 2007, A\&A, 470, L13

Drake, J. J., Ercolano, B., \& Swartz, D. A. 2008, ApJ, 678, 385

Favata, F., Reale, F., Micela, G., et al. 2000, A\&A, 353, 987

Favata, F., Flaccomio, E., Reale, F., et al. 2005, ApJS, 160, 469

Franciosini, E., Pillitteri, I., Stelzer, B., et al. 2007, A\&A, 468, 485

Getman, K. V., Feigelson, E. D., Broos, P. S., Micela, G., \& Garmire, G. P. 2008, ApJ, 688, 418

Giardino, G., Favata, F., Micela, G. \& Reale, F. 2004, A\&A, 413, 669

Giardino, G., Favata, F., Silva, B., et al. 2006, A\&A, 453, 241

Giardino, G., Favata, F., Pillitteri, I., et al. 2007, A\&A, 475, 891

Güdel, M., Linsky, J. L., Brown, A., \& Nagase, F. 1999, ApJ, 511, 405

Güdel, M., Audard, M., Reale, F., Skinner, S. L., \& Linsky, J. L. 2004, A\&A, 416, 713

Haisch, B. M. 1983, ASSL, IAU Colloq. 71: Activity in Red-Dwarf Stars, 102, 255

Kastner, J. H., Crigger, L., Rich, M., \& Weintraub, D. A. 2003, ApJ, 585, 878

Katsova, M. M., Livshits, M. A., \& Schmitt, J. H. M. M. 2002, ASPC, 277, 515

Kopp, R. A., \& Pneuman, G. W. 1976, SoPh, 50, 85

Kopp, R. A., \& Poletto, G. 1984, Sol. Phys., 93, 351

Maggio, A., Pallavicini, R., Reale, F., \& Tagliaferri, G. 2000, A\&A, 356, 627

Morrison, R., \& McCammon, D. 1983, ApJ, 270, 119

Nordon, R., \& Behar, E. 2007, A\&A, 464, 309 
Osten, R. S., Drake, S., Tueller, J., et al. 2007, ApJ, 654, 1052

Pan, H. C., Jordan, C., Makishima, K., et al. 1997, MNRAS, 285, 735

Pettersen, B. R. 1991, Mem. Soc. Astron. Ital., 62, 217

Poletto, G., Pallavicini, R., \& Kopp, R. A. 1988, A\&A, 201, 93

Reale, F., Betta, R., Peres, G., Serio, S., \& McTiernan, J. 1997, A\&A, 325, 782

Reale, F., Peres, G., \& Orlando, S. 2001, ApJ, 557,906

Reale, F. 2002, ASPC, 277, 103

Reale, F. 2003,AdSpR, 32, 1057

Reale, F., Güdel, M., Peres, G., \& Audard, M. 2004, A\&A, 416, 733

Reale, F. 2007, A\&A, 471, 271

Robrade, J., \& Schmitt, J. H. M. M 2005, A\&A, 435, 1073

Rucinski, S. M., \& Krautter, J. 1983, A\&A, 121, 217

Scholz, A., Coffey, J., Brandeker, A., \& Jayawardhana, R. 2007, ApJ, 662, 1254

Sciortino, S. 2008, MmSAI, 79, 192

Serio, S., Reale, F., Jakimiec, J., Sylwester, B., \& Sylwester, J. 1991, A\&A, 241, 197

Siess, L., Dufour, E., \& Forestini, M. 2000, A\&A, 358,593

Smith, R. K., Brickhouse, N. S., Liedahl, D. A., \& Raymond, J. C. 2001a, ApJ, 556, L91

Smith, R. K., Brickhouse, N. S., Liedahl, D. A., \& Raymond, J. C. 2001b, ASP Conf. Ser. 247: Spectroscopic Challenges of Photoionized Plasmas, 247, 161

Stauffer, J. R., Hartmann, L. W., \& Barrado y Navascues, D. 1995, ApJ, 454, 910

Stelzer, B., Burwitz, V., Audard, M., et al. 2002, A\&A, 392, 585
Stelzer, B., \& Schmitt, J. H. M. M. 2004, A\&A, 418,687

Stelzer, B., Flaccomio, E., Briggs, K., et al. 2007, A\&A, 468, 463

Sylwester, B., Sylwester, J., Serio, S., Reale, F., Bentley, R. D., \& Fludra, A. 1993, A\&A, 267, 586

Testa, P., Reale, F., Garcia-Alvarez, D., \& Huenemoerder, D. P. 2007, ApJ, 663, 1232

Testa, P., Drake, J. J., Ercolano, B., et al. 2008, ApJ, 675, L97

Tsujimoto, M., Feigelson, E. D., Grosso, N., et al. 2005, ApJS, 160, 503

Vaiana, G. S., Cassinelli, J. P., Fabbiano, G., et al. 1981, ApJ, 245, 163

van den Oord, G. H. J., Mewe, R., \& Brinkman, A. C. $1988, A \& A, 205,181$

van den Oord, G. H. J., \& Mewe, R. 1989, A\&A, 213,245

This 2-column preprint was prepared with the AAS LATEX macros v5.2. 